\title{
Modeling of diffusion of injected electron spins in spin-orbit coupled microchannels
}

\author{
Liviu P. Zârbo, ${ }^{1}$ Jairo Sinova, ${ }^{2,1}$ I. Knezevic, ${ }^{3}$ J. Wunderlich, ${ }^{4,1}$ and T. Jungwirth ${ }^{1,5}$ \\ ${ }^{1}$ Institute of Physics ASCR, v.v.i., Cukrovarnická 10, 16253 Praha 6, Czech Republic \\ ${ }^{2}$ Department of Physics, Texas A \& M University, College Station, Texas 77843-4242, USA \\ ${ }^{3}$ Department of Electrical and Computer Engineering, University of Wisconsin-Madison, Madison, Wisconsin 53706, USA \\ ${ }^{4}$ Hitachi Cambridge Laboratory, Cambridge CB3 OHE, United Kingdom \\ ${ }^{5}$ School of Physics and Astronomy, University of Nottingham, Nottingham NG7 2RD, United Kingdom \\ (Received 24 August 2010; revised manuscript received 19 October 2010; published 16 November 2010)
}

\begin{abstract}
We report on a theoretical study of spin dynamics of an ensemble of spin-polarized electrons injected in a diffusive microchannel with linear Rashba and Dresselhaus spin-orbit coupling. We explore the dependence of the spin-precession and spin-diffusion lengths on the strengths of spin-orbit interaction and external magnetic fields, microchannel width, and orientation. Our results are based on numerical Monte Carlo simulations and on approximate analytical formulas both treating the spin-dynamics quantum mechanically. We conclude that spin-diffusion lengths comparable or larger than the precession length occur (i) in the vicinity of the persistent spin helix regime for arbitrary channel width and (ii) in channels of similar or smaller width than the precession length, independent of the ratio of Rashba and Dresselhaus fields. For similar strengths of the Rashba and Dresselhaus fields, the steady-state spin-density oscillates or remains constant along the channel for channels parallel to the in-plane diagonal crystal directions. An oscillatory spin-polarization pattern tilted by $45^{\circ}$ with respect to the channel axis is predicted for channels along the main cubic crystal directions. For typical experimental system parameters, magnetic fields on the order of tesla are required to affect the spin-diffusion and spin-precession lengths.
\end{abstract}

DOI: 10.1103/PhysRevB.82.205320

PACS number(s): 75.76. +j, 71.70.Ej, 61.43.Bn

\section{INTRODUCTION}

Spin-orbit (SO) coupling in vacuum is a relativistic effect in which the magnetic moment of a moving electron couples to an external electric field. The effect can be explained by recalling that the moving magnetic moment is seen in the laboratory frame as both magnetic and electric dipole moment and the electric dipole component couples to the external electric field. The correct magnitude of the SO coupling term can be derived using the Dirac equation for the moving particle. Owing to the band structure, the SO coupling for electrons in solids can be enhanced by orders of magnitude with respect to the value computed in vacuum. This makes the SO coupling-based effects experimentally accessible and enables the use of SO coupling as a tool for purely electrical generation and manipulation of spins in devices. ${ }^{1-4}$

The prototype spintronic device using SO coupling as a spin control tool is the Datta-Das transistor. ${ }^{5}$ It consists of a SO-coupled-channel connected to spin-polarized source and drain electrodes. Inside the channel, the electron undergoes coherent spin rotations under the influence of the SO field which can be tuned electrically by an external gate. However, the simplicity of the Datta-Das concept is deceptive whenever the channel is not one-dimensional. The main problem concerning spin transport in the channel of a DattaDas device is that on one hand, the SO coupling strength in the channel has to be large enough to enable control of the electron spin. On the other hand, however, a large SO coupling can lead to a faster spin relaxation via D'yakonov-Perel ${ }^{6}$ mechanism than the electron dwell time in the channel if the channel is not one dimensional or ballistic. To overcome the difficulty, it was proposed ${ }^{7,8}$ to exploit the symmetry arising from the interplay of Rashba $^{9}$ and
Dresselhaus ${ }^{10} \mathrm{SO}$ fields in the two-dimensional electron gas (2DEG) formed in semiconductor heterostructures. This proposal has opened a way to the Datta-Das transistor operating in a nonballistic regime. ${ }^{7,8,11}$

SO fields in the 2DEG act as momentum-dependent magnetic fields that couple to the electronic magnetic moment. Impurities, phonons, or crystalline defects can scatter the electrons which changes their momenta and, therefore, changes the effective SO-induced magnetic field acting on the electron spin. Individual electron spins in the channel acquire different phases with respect to each other, resulting in the relaxation of the total spin. This is the qualitative picture of the D'yakonov-Perel relaxation.

The idea behind the nonballistic Datta-Das spin transistor is that one could tune the Rashba and Dresselhaus SO coupling strengths to be equal, e.g., via gate voltage. ${ }^{12}$ In this case, the orientation of the total Rashba-Dresselhaus SO field is independent of momentum and is parallel to one of the in-plane diagonal axes (that can be either [110] or [110] depending on the relative sign of the Rashba and Dresselhaus fields) in the (001) plane of the 2DEG in a cubic semiconductor. The amplitude of the SO field depends only on the momentum component perpendicular to the direction of the SO field. ${ }^{7,13}$ This can lead to a path-independent spin precession of individual electron spins and thus to a suppression of the spin relaxation, in 2DEG channels oriented perpendicular to the linear Rashba-Dresselhaus SO field. Moreover, the Hamiltonian exhibits the U(1) symmetry which means that an in-plane spin state parallel to this SO field direction is infinitely long lived. This state will be dephased if the cubic Dresselhaus term is present in the system. ${ }^{14-16}$ Randomness in the SO coupling induced by remote impurities would cause additional spin relaxation. ${ }^{17}$ Nevertheless, infinite spin 
lifetimes are still possible in SO-coupled 2DEGs if the spatially varying SO field can be described as a pure gauge and, thus, removed by a gauge transformation. ${ }^{18}$

Furthermore, it was shown ${ }^{13}$ that the many-electron system whose individual particles are described by the above U(1) symmetric single-particle Hamiltonian displays a SU(2) symmetry which is robust against both spin-independent disorder and electron-electron interactions. Owing to this symmetry, a collective spin state excited at a certain wave vector would have an infinite lifetime. Such a state is called the persistent spin helix ${ }^{13}$ (PSH) and it has already been observed in transient spin grating experiments. ${ }^{19,20}$

In another recent experiment, ${ }^{21}$ spin-polarized current passing through a micrometer-size 2DEG channel has been detected by measuring the SO coupling-induced Hall signal. This is called the spin-injection-Hall effect (SIHE). The fact that the SIHE observed in a diffusive channel is robust against disorder and temperature effects and that the estimated Rashba and Dresselhaus SO couplings are similar in the 2DEG system employed in the experiment leads to the question whether the PSH physics is relevant to this transport experiment.

In this paper, we investigate theoretically spin dynamics of electrons in the 2DEG channel in the PSH regime as well as in regimes of different Rashba and Dresselhaus SO field strengths. In the context of the above SIHE experiment we point out in particular that spin-diffusion lengths comparable to the spin-precession length occur in channels whose widths are smaller or comparable to the spin-precession length, regardless of the ratio between the Rashba and Dresselhaus SO coupling strengths. This is one of the several conclusions of the calculations presented below which consider the dependence of the spin-diffusion characteristics on experimentally relevant system parameters such as the strengths of SO and external magnetic fields, microchannel width, and orientation.

In our calculations we employ the noninteracting electron approximation $^{22,23}$ and consider the diffusive regime in which the SO splitting is much smaller than the energy-level broadening due to disorder scattering, $\Delta_{\text {SO }} \ll \hbar / \tau$. In this approach, momentum and position of electrons can be treated as classical variables. We emphasize that the direct correspondence mentioned above between the suppressed spin relaxation in the single-particle transport problem and the collective PSH state is valid in this diffusive regime. Here the group velocity of an electron in the Rashba-Dresselhaus 2DEG can be approximated by its momentum divided by the mass. The spin-precession angle of such a particle depends only on the distance traveled along the direction perpendicular to the SO field. ${ }^{13}$ The resulting spin-density pattern of an ensemble of injected electron spins then coincides with the spin-density pattern of the PSH spin wave. The expression of velocity of SO-coupled electrons contains terms proportional to SO coupling strength. For example, the velocity along [110] direction of Rashba and Dresselhaus SO-coupled electrons in the PSH regime $(\alpha=-\beta)$ is $\mathbf{v}_{\mathbf{1 1 0}}=\hbar \mathbf{k}_{\mathbf{1 1}} / m^{*} \pm 2 \beta / \hbar$, where $\alpha=-\beta$ are the Rashba and Dresselhaus SO coupling strengths. This means that in the opposite limit of strong SO coupling and weak disorder, the velocity and momentum are

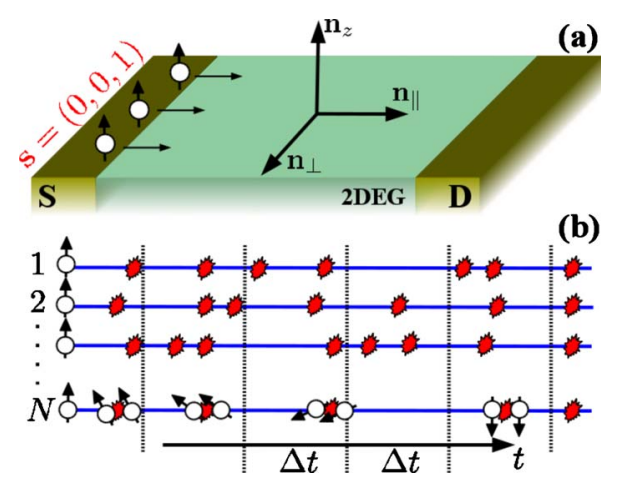

FIG. 1. (Color online) (a) Representation of the our model device. Spin- $\uparrow$ polarized particles are injected from the source electrode in a Rashba and Dresselhaus SO-coupled channel. (b) Schematic depiction of the EMC method. The time evolution of each particle belonging to the ensemble is sampled at equal intervals $\Delta t$ called subhistories. The particle spin processes in the SO field during the free flight time but is unaffected by collisions.

not simply proportional to each other and the direct link is lost between the one particle and collective physics in the regime of equal or similar Rashba and Dresselhaus field strengths.

The paper is organized as follows. In Sec. II we introduce our method and discuss our approximations. In Sec. II A we outline the features of the Monte Carlo method we use in our simulations. In Sec. II B we discuss the single-particle evolution of quantum spin in SO-coupled 2DEG and its dependence on crystalline direction of propagation, external magnetic field, and interplay of Rashba and Dresselhaus SO couplings. In Sec. III we show how the steady-state spindensity distribution of an ensemble of electrons in the channel is affected by varying the above parameters. Section IV gives the main conclusions of our work.

\section{THEORETICAL MODEL}

We are interested in the spin dynamics in the 2DEG channel of an experimentally relevant spintronic model device which is schematically depicted in Fig. 1(a). The typical device is of a few micrometers in size and this is considerably larger than the Fermi wavelength in the 2DEG channel. It means that the quantum interference effects on the orbital motion of electrons can be neglected. In other words, it is sufficient to solve Boltzmann-transport equation (BTE) for this system, rather than use a fully quantum-mechanical treatment such as Keldysh formalism. On the other hand, we cannot neglect quantum mechanics of the spin dynamics since the typical spin-precession length in experiments ${ }^{21}$ ranges from a few hundreds of nanometers to a few microns. Therefore, we employ the ensemble Monte Carlo ${ }^{24-27}$ (EMC) method which is a well-established tool in semiconductordevice simulations and can be extended to include spin coherence $^{28}$ in a micrometer-size device. The EMC method offers a way to solve BTE that is beyond the reach of driftdiffusion models. Drift-diffusion models must rely on various approximations in order to avoid the tremendous mathematical difficulties arising in BTE. Treatment of nonlinear 
terms, inclusion of different scattering mechanisms or of dissipation effects require drastic approximations so that the result of the drift-diffusion calculation might not reflect anymore the features of the theoretical model but rather those of the mathematical approximations. By contrast, including disorder, dissipation, temperature, transient, or nonlinear effects in EMC simulations is straightforward and does not require further approximations. In fact, state-of-the-art EMC simulations are often used to test the validity of the drift-diffusion models. Inclusion of a various range of effects in the EMC is done without significant changes in the computational complexity, thus, making it more suitable for device simulation than other powerful quantum-mechanical techniques such as nonequilibrium Green's function (NEGF) method. For example, including dissipative effects in EMC has only a minor impact on the calculation complexity while in the case of NEGF method it can reduce the size of computationally accessible systems from a few hundreds to a few tens of nanometers.

In our calculations, we make the following approximations: (i) electron orbital degrees of freedom are described by classical momentum and position and the spin degree of freedom by quantum-mechanical spin-density matrix. This semiclassical approximation is justified by the diffusive regime we consider. In this regime we can approximate the electron velocity by momentum divided by mass. (ii) Interactions between electrons are neglected. (iii) We consider only shortrange impurity scattering. (iv) Temperature enters our simulations only through the Fermi-Dirac distribution function. (v) For simplicity, we neglect the electrostatics of the channel. This is justified since we are primarily interested in the spin dynamics of electrons. A small electric field present in the channel is not expected to have an important influence on the spin-precession pattern of the electronic system.

In the next two sections we briefly outline the spindependent EMC method and analyze the motion of a single particle in the SO field.

\section{A. Spin dependent Monte Carlo}

Electrons in the channel, shown in Fig. 1(a), can be modeled as an ensemble of $N$ noninteracting particles. In the EMC method, we track the individual motion of each particle in the ensemble and we use the data to calculate an approximate particle distribution in-phase space. As shown in Fig. 1(b), we divide the time of simulation in small time intervals $\Delta t$ called subhistories. During the subhistory, a particle moving in electromagnetic and SO fields in the channel can be randomly scattered by impurities and, in general, also by phonons or other scattering mechanisms that are present in the channel. The time between collisions, called the "free flight time," is randomly generated and depends on the scattering rate corresponding to each type of collision. ${ }^{25}$ Figure 1(b) gives an intuitive picture of the time evolution of the ensemble of electrons. The semiclassical particle is described by its position $\mathbf{r}(t)$ and momentum $\mathbf{k}(t)$. As it was recently shown, ${ }^{22,28}$ we can treat spin-dependent phenomena if, in addition to the semiclassical variables, we consider that each particle is described by a $2 \times 2$ spin-density matrix $\hat{\rho}(t)$. The spin-polarization vector is then given by $\mathbf{s}=\operatorname{Tr}[\hat{\rho} \sigma]$. The propagation of a particle during the free flight is described by the equations of motion for its attached dynamical variables

$$
\begin{gathered}
m^{*} \frac{d^{2} \mathbf{r}}{d t^{2}}=-e\left[\mathbf{E}+\mathbf{v}_{\mathbf{k}} \times \mathbf{B}\right], \\
\mathbf{v}_{\mathbf{k}}=\frac{1}{\hbar} \nabla_{\mathbf{k}} E_{\mathbf{k}} \approx \frac{\hbar \mathbf{k}}{m^{*}}, \\
\hat{\rho}(t+\delta t)=e^{-i / \hbar \hat{H}_{\mathrm{spin}}(\mathbf{k}) \delta t} \hat{\rho}(t) e^{i / \hbar \hat{H}_{\mathrm{spin}}(\mathbf{k}) \delta t},
\end{gathered}
$$

which must be integrated together to find the particle time evolution during free flights. Here, $\mathbf{v}_{\mathbf{k}}$ is the particle velocity, $\mathbf{E}$ and $\mathbf{B}$ are the electric and magnetic fields, $m^{*}$ is the effective mass of the particle, and $E_{\mathbf{k}}$ is the electronic band dispersion in the 2DEG. Note that the approximation in Eq. (1b) means that we neglect any influence of the SO coupling on the trajectory of the semiclassical particle. ${ }^{29}$ While Eqs. (1a) and (1b) describe a semiclassical electron propagating in a solid, Eq. (1c) describes the quantum-mechanical evolution of its spin during short time $\delta t$ which is controlled by the spin-dependent part of the Hamiltonian, $\hat{H}_{\text {spin }}$. This Hamiltonian includes the internal SO field and the external magnetic field, $\hat{H}_{\text {spin }}=\hat{H}_{\mathrm{SO}}+\hat{H}_{\mathrm{Z}}$.

At the end of each subhistory we calculate the ensemble averaged quantities of interest such as currents, charge, and spin densities. After the simulation converged and the system is in steady state we can use the subsequent subhistories to compute time averaged values for the physical quantities. In our case, the system is in steady state when the flux of electrons through the drain electrode becomes constant.

\section{B. Spin dynamics in Rashba-Dresselhaus field}

The electron gas in the heterostructure can be modeled by the Rashba and Dresselhaus SO-coupled Hamiltonian

$$
\hat{H}=\frac{\hat{\mathbf{p}}^{2}}{2 m^{*}}+\frac{\alpha}{\hbar}\left(\hat{p}_{y} \sigma_{x}-\hat{p}_{x} \sigma_{y}\right)+\frac{\beta}{\hbar}\left(\hat{p}_{x} \sigma_{x}-\hat{p}_{y} \sigma_{y}\right) .
$$

Here $\beta$ is the Dresselhaus SO coupling which, for simplicity, is kept constant in our simulations, $\alpha$ is the experimentally adjustable ${ }^{12,30}$ Rashba parameter, $m^{*}$ is the effective mass of the 2DEG, and $\sigma_{x}, \sigma_{y}$, and $\sigma_{z}$ are the Pauli matrices. The crystalline axes labeled $x, y$, and $z$ correspond to the [100], [010], and [001] directions, respectively, and the 2DEG lies in the $x y$ plane. We are ignoring the cubic Dresselhaus terms since the linear terms are dominant for not too high-carrier concentrations. The effect of the magnetic field is included by making the substitution $\mathbf{p} \rightarrow \mathbf{p}-\boldsymbol{e \mathbf { A }}$ in Hamiltonian (2) and by adding the Zeeman term,

$$
\hat{H}_{\mathrm{Z}}=-\frac{1}{2} g \mu_{\mathrm{B}} \mathbf{B} \cdot \sigma=-\frac{1}{2} g \mu_{\mathrm{B}}\left(B_{\|} \sigma_{\|}+B_{\perp} \sigma_{\perp}+B_{z} \sigma_{z}\right),
$$

where $\mathbf{B}$ is the magnetic field strength, $\mathbf{A}$ is the corresponding vector potential, $g$ is the $g$ factor, and $\mu_{\mathrm{B}}$ is the Bohr magneton. We wrote the Zeeman Hamiltonian in terms of the magnetic field components $B_{\|}$which is parallel to the trans- 
port direction in the channel, $B_{\perp}$ which is the in-plane magnetic field component perpendicular to the current direction, and $B_{z}$ which is the out-of-plane component of the magnetic field. The unit vectors corresponding to in-plane axes parallel and perpendicular to the transport direction are labeled by $\mathbf{n}_{\|}=(a, b, 0)$ and $\mathbf{n}_{\perp}=(b,-a, 0)$ while $\mathbf{n}_{z}=(0,0,1)$ corresponds to the $z$ axis. For example, if the electronic transport is along [110] axis, $a=1 / \sqrt{2}$ and $b=-1 / \sqrt{2}$. Using this notation we can express the spin matrices as $\sigma_{\|}=\mathbf{n}_{\|} \boldsymbol{\sigma}=a \sigma_{x}+b \sigma_{y}$ and $\sigma_{\perp}=\mathbf{n}_{\perp} \boldsymbol{\sigma}=b \sigma_{x}-a \sigma_{y}$. Note that the above Hamiltonian (3) does not take into account the change in the effective mass or $g$ factor in the 2DEG as a result of applying magnetic field. ${ }^{31}$

In what follows, we derive the spin-precession length of an electron propagating in a straight line along an arbitrary direction in the SO-coupled 2DEG. As in the spin-dependent EMC approach described in the previous section, the electron is a point particle whose spin rotates coherently under the influence of a weak SO field and the applied external magnetic field. We consider a spin- $\uparrow$ electron (spin parallel to $+\hat{z}$ direction) injected along an arbitrary direction $\mathbf{n}_{\|}$ $=(a, b, 0)$ and subject to both $\mathrm{SO}$ and magnetic fields. The electron spin is described by the spin-density matrix $\rho$ $=\frac{1}{2}\left(\mathbf{I}_{2}+\mathbf{s} \boldsymbol{\sigma}\right)$, where $\mathbf{s}=\left(s_{x}, s_{y}, s_{z}\right)$ is the spin-polarization vector and $\mathbf{I}_{2}$ is the $2 \times 2$ identity matrix. Initially, the electron has spin $\uparrow$, so its polarization vector is $\mathbf{s}=(0,0,1)$ and the spin-density matrix is $\rho_{0}=\frac{1}{2}\left(\mathbf{I}_{2}+\sigma_{z}\right)$.

Next, we rewrite the SO part of Hamiltonian (2) as

$$
\hat{H}_{\mathrm{SO}}=\Omega_{x} \sigma_{x}+\Omega_{y} \sigma_{y}+\Omega_{z} \sigma_{z}
$$

with $\Omega_{x}=\alpha k_{y}+\beta k_{x}, \Omega_{x}=\left(\alpha k_{x}+\beta k_{y}\right)$, and $\Omega_{z}=0$. We label the unit vector parallel to $\boldsymbol{\Omega}=\left(\Omega_{x}, \Omega_{y}, \Omega_{z}\right)$ by $\mathbf{h}=\left(h_{x}, h_{y}, h_{z}\right)$. After a short-time step $\delta t$ during which the momentum is considered constant, we obtain with the aid of Eq. (1c)

$$
\rho(\delta t)=\frac{1}{2} \mathbf{I}_{2}+\frac{1}{2} \cos (2 P \delta t) \sigma_{z}-\frac{1}{2} \sin (2 P \delta t)\left(h_{x} \sigma_{y}-h_{y} \sigma_{x}\right),
$$

where $P=\frac{1}{\hbar} \sqrt{\Omega_{x}^{2}+\Omega_{y}^{2}}$. In order to study the single-particle spin precession we consider that the electron momentum along the transport direction is constant. Such assumption is true as long as there is no transverse external electric field and no out-of-plane magnetic field. The condition that the spin flips during the motion of the electron is $2 P t^{\uparrow \rightarrow \downarrow}=\pi$, as seen from Eq. (5). The spin-precession length along the transport direction $\mathbf{n}_{\|}=(a, b, 0)$ is computed as $L_{\mathrm{ab} 0}^{\uparrow \rightarrow}$ $=\mathbf{v}_{\mathbf{k}} t^{\uparrow \rightarrow \downarrow}$. (Recall that $\mathbf{v}_{\mathbf{k}} \approx \hbar \mathbf{k} / m^{*}$ in the diffusive, weak SOcoupling regime.) Considering the effects of the in-plane magnetic field, we obtain for the spin-precession length

$$
L_{\mathrm{ab} 0}^{\uparrow \rightarrow}=\frac{\pi \hbar^{2}}{2 m^{*} \sqrt{\left(\alpha b+\beta a+\frac{1}{2} g \mu_{\mathrm{B}} \frac{B_{\|} a+B_{\perp} b}{k}\right)^{2}+\left(-\alpha a-\beta b+\frac{1}{2} g \mu_{\mathrm{B}} \frac{B_{\|} b+B_{\perp} a}{k}\right)^{2}}}
$$

By applying the spin-precession length formula (6) we can gain an intuitive understanding of the spin dynamics in the SO-coupled heterostructure. Of particular interest is the case of $\alpha=-\beta$ and [1 10$]$ channel orientation (or $\alpha=\beta$ and [110] orientation) in which the PSH symmetry is present. In this case, the spin precession depends only on the distance traveled by electrons along the channel. From Eq. (6) we can immediately see that for $\alpha=-\beta$ and electron propagating along the $[1 \overline{1} 0]$ direction, the spin-precession length is the shortest while for spin propagating along the [110] direction it is infinite.

\section{DISCUSSION OF THE EMC SIMULATIONS}

We now employ the spin-dependent EMC method outlined in Sec II A to numerically simulate spin dynamics in the microchannel illustrated in Fig. 1(a). Spin- $\uparrow$ electrons are injected from the source electrode and propagate in the SO field of the disordered 2DEG. Electrons that reach the microchannel edge are reflected back with unchanged spin. An electron that re-enters the source or exits the drain is erased and replaced by a new spin- $\uparrow$ electron injected from the source.
We choose the 2DEG parameters that correspond to the GaAs 2DEG of Ref. 21. Our channel length is $L \approx 3 \mu \mathrm{m}$ and the width will take values both smaller and larger than the spin-precession length which is on the order of a few hundred nanometers. Temperature of the electron ensemble in all simulations is $300 \mathrm{~K}$. Electron-phonon scattering is neglected. Disorder in the system is due to randomly placed pointlike spinless impurities. The electron mean free path is $26 \mathrm{~nm}$. The electronic density of the 2DEG system is $n_{e}$ $=2.5 \times 10^{12} \mathrm{~cm}^{-2}$. The corresponding Fermi wavelength is $\lambda_{F} \approx 8 \mathrm{~nm}$. The Dresselhaus spin-orbit coupling is kept constant during simulations at $\beta=-2.0 \times 10^{-12} \mathrm{eV} \mathrm{m}$.

The electron ensemble consists of $N=130000$ electrons. We run the simulation until the system reaches steady state and after that we use the last 2000 time steps to calculate time averaged spin densities. The spin densities are normalized to the number of particles present in each grid cell such that the spin of a cell containing all spin- $\uparrow$ particles is 1 . In what follows, we show how the spin-density distribution $\left\langle S_{z}(\mathbf{r})\right\rangle$ in the channel is affected by changes in the width of the channel, the crystalline axis along which the transport takes place, and strength of Rashba SO coupling and magnetic fields. 


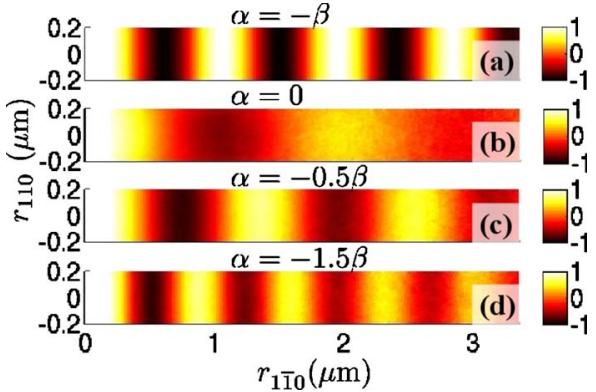

FIG. 2. (Color online) Spin-density distribution $\left\langle S_{z}(\mathbf{r})\right\rangle$ in a [1] 10$]$-oriented 2DEG channel with fixed Dresselhaus SO coupling $\beta=-2.0 \times 10^{-12} \mathrm{eV} \mathrm{m}$ for different values of Rashba SO coupling (a) $\alpha=-\beta$, (b) $\alpha=0$, (c) $\alpha=-0.5 \beta$, and (d) $\alpha=-1.5 \beta$. The light color refers to spin $\uparrow$. The distance between the successive maxima of the spin distribution corresponds to twice the spin-precession length computed from Eq. (6). The spin-diffusion length is infinite in (a) while in all the other cases it exceeds the spin-precession length, as expected in narrow channel.

In general, the spin polarization along the channel is randomized due to the D'yakonov Perel spin dephasing mechanism which is dominant in GaAs heterostructures. This effect is visible, e.g., in Fig. 2(b). The spin-diffusion length depends on the parameters of the microchannel. In the limiting case of equal Rashba and Dresselhaus SO coupling strengths and, e.g., $\alpha=-\beta$, the PSH symmetry ${ }^{13}$ arises in the [110]-oriented 2DEG channel and the oscillatory dependence of $\left\langle S_{z}(\mathbf{r})\right\rangle$ on the coordinate along the channel is undamped, as shown in Fig. 2(a). In this case spin orientations of injected electrons are not randomized by scattering. The spin-diffusion length is infinite and the spin-precession length is given exactly by Eq. (6). In Figs. 2(c) and 2(d) we show that the PSH regime is robust against sizable changes in the $\alpha / \beta$ ratio. $^{23}$

We next proceed to channels which are not oriented along the $[1 \overline{1} 0]$ direction. In Fig. 3 we compare results for the [100]- and [110]-oriented channels with the [1ํㅣ channel, assuming $\alpha=-\beta$. Figure 3(a) shows that the spin precesses fastest for the channel oriented along the [1 $\overline{1} 0]$ direction while Fig. 3(b) shows no spin precession for the [110]oriented channel, consistent with Eq. (6). The result in Fig. 3 (c) for the channel oriented along the [100] axis is less obvious, however, we can still use the spin precession formula (6) to understand the $45^{\circ}$ rotated oscillatory pattern for this channel direction. Since the orientation of the spin depends on the distance the particle travels along the [1 $\overline{1} 0]$ direction, we expect that a pattern formed by averaging the spin densities along a [110]-oriented line would repeat itself with a $2 L_{1 \overline{1} 0}^{\uparrow \rightarrow \uparrow}$ period along the $[1 \overline{1} 0]$ line. As sketched in Fig. 3 , we can follow individual trajectories of two electrons injected from the source. Let us consider two particles and connect them by the relative position vector $\mathbf{d}$ whose length $d$ is the initial distance between particles. We can decompose

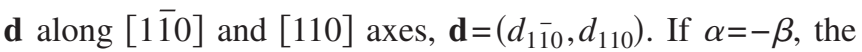
difference between the spin directions of the two particles when they meet inside the channel is given by $d_{110}$. The spin

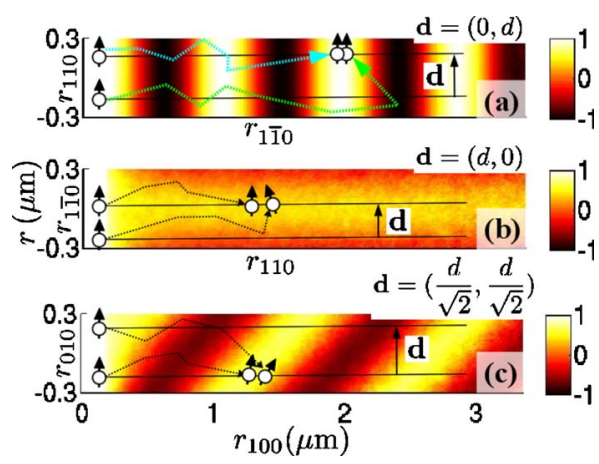

FIG. 3. (Color online) Spin-density distribution $\left\langle S_{z}(\mathbf{r})\right\rangle$ in the 2DEG channel with $\alpha=-\beta$ for various orientation of the transport axis: (a) $[1 \overline{1} 0]$, (b) [110], and (c) [100]. In the PSH regime, the relative spin orientations of particles injected at the source depends only on the initial distance between them along the $[1 \overline{1} 0]$ direction, i.e., the first component of the relative position vector of the two particles $\mathbf{d}=\left(d_{1 \overline{1}}, d_{110}\right)$. In panel (a) that distance is $d_{1 \overline{1}}=0$, resulting in no spin dephasing, in panel (b) is $d_{1 \overline{10}}=d$, and in panel (c) it is $d_{1 \overline{10}}=d / \sqrt{2}$, where $d$ is the initial distance between the two particles. Therefore, the spin relaxation in the [110]- and [100]oriented channels depends on the channel width.

coherence of the electron ensemble is therefore partially lost because of the initial distribution of $d_{110}$ 's of the injected electrons. From this it is apparent that the spin-diffusion length scales with the ratio of the precession length to the channel width. We emphasize that all these arguments are independent of the mean free path. Indeed, we would obtain the same steady-state spin-density distribution if the channel in Fig. 3 were ballistic.

The dependence of the spin-diffusion length in our ensemble of electrons on the channel width is further quantified in Fig. 4. For the [1 $\overline{1} 0]$-oriented channel the spin-diffusion length is infinite as long as $\alpha=-\beta$ and it decreases with increasing width of the channel when Rashba and Dresselhaus coupling strengths are not equal, as shown in Fig. 4(a). ${ }^{22,32}$ For $\alpha=-\beta$, the spin-diffusion length is finite for channel orientations different from [1히 direction and it again decreases with increasing channel width. This is illustrated in Fig. 4(b) for the [110]-oriented microchannel and in Fig. 4(c) for the [100] channel.

We now provide a more detailed understanding of the numerical spin-density patterns obtained by the EMC simulations, focusing on the $\alpha=-\beta$ case and the [110]-oriented microchannels. For fixed and equal Rashba and Dresselhaus coupling strengths $(\alpha=-\beta)$, the spin orientation of an individual particle depends only on the distance from the injection point along the $[1 \overline{1} 0]$ direction. The spin-density pattern of an ensemble of particles starting from a given point depends only on the strength of the SO coupling. Our ensemble averaging procedure amounts to summing up all spin-density patterns of particles starting from different points along the source-channel interface. We use this idea to explain the spin-density distribution obtained for the [110]-oriented channel of two different widths, as shown in Fig. 5. The width of the channel in Fig. 5(c) is equal to the spinprecession length $L_{110}^{\uparrow \rightarrow \uparrow}$. All spins starting at the source at 


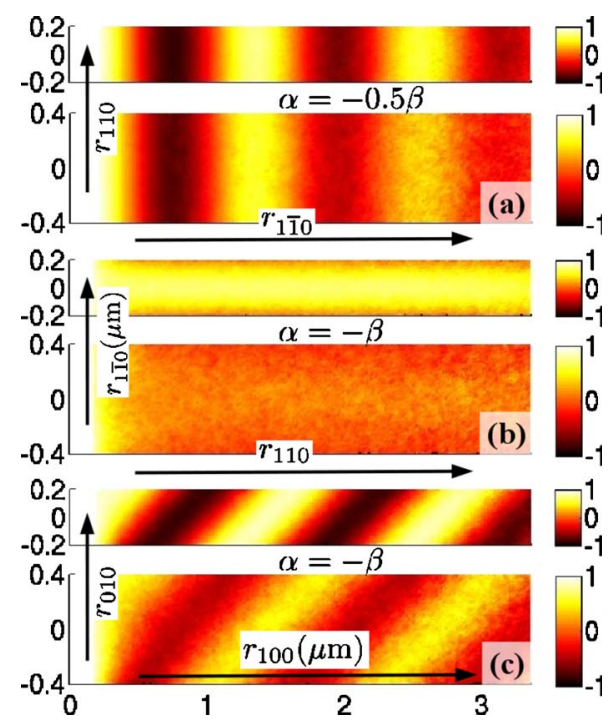

FIG. 4. (Color online) Spin-density distribution $\left\langle S_{z}(\mathbf{r})\right\rangle$ in the channel as a function of its width for (a) $\alpha=-0.5 \beta$ and [1 $1 \overline{1} 0]$ injection, (b) $\alpha=-\beta$ and [100]-oriented channel, and (c) $\alpha=-\beta$ and [110]-injection direction. As expected from theory, the larger channel widths lead shorter spin-diffusion lengths.

point A shown in Fig. 5(a) generate a spin-density pattern illustrated in the corresponding column $\mathrm{A}$ in the figure. The spin-density pattern generated by a spin starting at point $B$ is the same, only shifted by the distance between A and B along the $[1 \overline{1} 0]$ direction. All the other spin-density patterns are shifted in the same manner. We can assign numbers to the projection of spin along $z$ direction for each spin-density pattern, as shown in Fig. 5(b). Summing up these numbers we obtain qualitatively the same transverse profile of the spin density in the channel as in the EMC simulation. This gives an intuitive explanation of the reduced mean spin polarization along the channel edges for channel width smaller or equal to $L_{\mathrm{SO}}$ seen in Fig. 5(c). We can use the same proce-

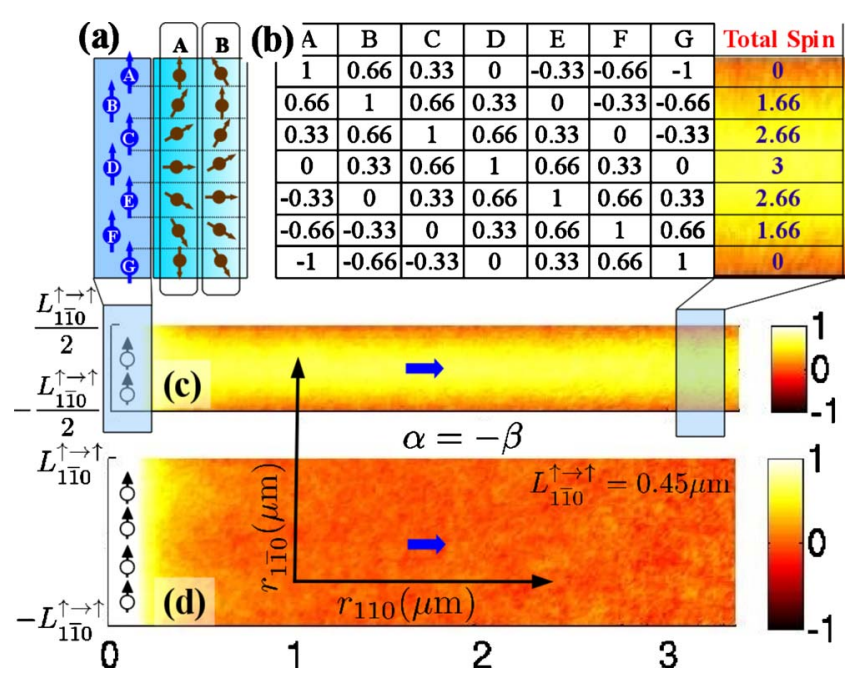

FIG. 5. (Color online) $[(a)$ and (b)] Pictorial explanation of the spin-density patterns in the $\alpha=-\beta$ regime for electrons injected along a [110]-oriented channel of width (c) $L_{1 \overline{1} 0}^{\uparrow \rightarrow \uparrow}$ and (d) $2 L_{1 \overline{1} 0}^{\uparrow \rightarrow \uparrow}$.

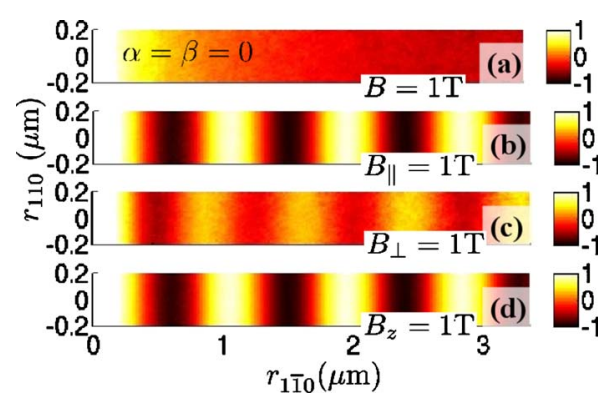

FIG. 6. (Color online) Spin-density distribution $\left\langle S_{z}(\mathbf{r})\right\rangle$ in magnetic field for the SO-coupled 2DEG channel oriented along [1 $\overline{1} 0]$ direction. The four panels show (a) Hanle effect for $1 \mathrm{~T}$ in-plane field and no SO coupling, spin precession for (b) $\alpha=-\beta$ and inplane magnetic field $B_{\|}=1 \mathrm{~T}$, (c) $\alpha=-\beta$ and in-plane magnetic field $B_{\perp}=1 \mathrm{~T}$, and (d) $\alpha=-\beta$ and out-of-plane magnetic field $B_{z}=1 \mathrm{~T}$.

dure to explain the randomization of spins in the entire cross section of a wider, [110]-oriented channel shown in Fig. 5(d).

As apparent from the one particle formula (6), the magnetic field effect on the spin dynamics depends on the magnitude of the electron momentum and, therefore, on the electron density in the channel. As shown in Fig. 6(a), the momentum dependence leads to faster randomization of the spin-density distribution in the channel than in the momentum independent case of Dresselhaus coupled 2DEG channel shown in Fig. 2(b). We will first analyze the effect of the in-plane magnetic field on spin precession. Our calculations in Fig. 6 show that in the $\alpha=-\beta$ case and for the $[1 \overline{1} 0]$-oriented channel, the spin density is only slightly affected by the in-plane magnetic field parallel to the channel direction even at magnitudes on the order of tesla. In-plane magnetic field of the same magnitude but perpendicular to the channel direction has a sizable effect on the electronic spin-density pattern. To understand the dependence on the in-plane field orientation we rewrite Eq. (6) for $\alpha=-\beta$ and for the $[1 \overline{1} 0]$ channel,

$$
L_{1 \overline{10}}^{\uparrow \rightarrow \downarrow}=\frac{\pi \hbar^{2}}{2 m^{*} \sqrt{4 \beta^{2}-\beta g \mu_{\mathrm{B}} \frac{B_{\perp}}{k}+\frac{1}{4} g^{2} \mu_{\mathrm{B}}^{2} \frac{B_{\perp}^{2}+B_{\|}^{2}}{k^{2}}} .}
$$

From Eq. (7) we see that for fields $\sim 1 \mathrm{~T}$, the magnetic field component $B_{\perp}$ affects the spin precession since the Zeeman splitting is comparable to the Dresselhaus spin splitting at the Fermi level. On the other hand, at higher magnetic fields, the quadratic terms in magnetic field present in the denominator of Eq. (7) will dominate the linear term and the effect of magnetic field becomes independent of its orientation. At these high fields, the spin-diffusion length is limited primarily by the magnetic field.

\section{SUMMARY}

We used the spin-dependent EMC method to simulate spin dynamics of an ensemble of electrons in the 2DEG channel with SO coupling. Our calculations were done in the diffusive regime in which there is a correspondence between 
the long spin-diffusion length of an ensemble of noninteracting electrons and the collective PSH state.

Our numerical simulations and qualitative analytical considerations show that the spin-precession pattern and the spin-diffusion length in the channel with equal strengths of the Rashba and Dresselhaus SO fields depends only on geometric factors, i.e., on the channel orientation and width. The presence of magnetic field in the channel suppresses the spin-diffusion length. However, for the experimentally relevant system parameters the fields magnitude must be on the order of a few tesla for the effect to be observable. The presence of the PSH symmetry yields an oscillatory spindensity pattern with infinite spin-diffusion length in [1] 0$]$-oriented channels of an arbitrary width. The spindiffusion length is still comparable to the precession length for any ratio of the Rashba and Dresselhaus fields as long as the channel width is comparable or smaller than the spinprecession length. These predictions are independent of the scattering mean free path.

\section{ACKNOWLEDGMENTS}

We acknowledge support from EU under Grant No. FP7215368 SemiSpinNet, from Czech Republic under Grants No. AV0Z10100521, No. KAN400100652, and No. LC510, and Praemium Academiae, and from NSF-MRSEC under Grants No. DMR-0820414 and No. DMR-0547875, and SWAN-NRI. L.P.Z. would like to thank Max Fischetti for correspondence.
${ }^{1}$ I. Žutić, J. Fabian, and S. Das Sarma, Rev. Mod. Phys. 76, 323 (2004).

${ }^{2}$ J. Fabian, A. Matos-Abiague, C. Ertler, P. Stano, and I. Zutic, Acta Phys. Slov. 57, 565 (2007).

${ }^{3}$ J. Sinova and A. H. MacDonald, in Spintronics, Semiconductors and Semimetals Vol. 82, edited by T. Dietl, D. D. Awschalom, M. Kaminska, and H. Ohno (Elsevier, New York, 2008), p. 45.

${ }^{4}$ M. Wu, J. Jiang, and M. Weng, Phys. Rep. 493, 61 (2010).

${ }^{5}$ S. Datta and B. Das, Appl. Phys. Lett. 56, 665 (1990).

${ }^{6}$ M. I. D'yakonov and V. I. Perel, Sov. Phys. Solid State 13, 3023 (1971).

${ }^{7}$ J. Schliemann, J. C. Egues, and D. Loss, Phys. Rev. Lett. 90, 146801 (2003).

${ }^{8}$ X. Cartoixà, D. Z.-Y. Ting, and Y.-C. Chang, Appl. Phys. Lett. 83, 1462 (2003).

${ }^{9}$ Y. Bychkov and E. I. Rashba, JETP Lett. 39, 78 (1984).

${ }^{10}$ G. Dresselhaus, Phys. Rev. 100, 580 (1955).

${ }^{11}$ K. C. Hall, W. H. Lau, K. Gündogdu, M. E. Flatté, and T. F. Boggess, Appl. Phys. Lett. 83, 2937 (2003).

${ }^{12}$ J. Nitta, T. Akazaki, H. Takayanagi, and T. Enoki, Phys. Rev. Lett. 78, 1335 (1997).

${ }^{13}$ B. A. Bernevig, J. Orenstein, and S.-C. Zhang, Phys. Rev. Lett. 97, 236601 (2006).

${ }^{14}$ T. D. Stanescu and V. Galitski, Phys. Rev. B 75, 125307 (2007).

${ }^{15}$ J. L. Cheng and M. W. Wu, J. Appl. Phys. 99, 083704 (2006).

${ }^{16}$ J. L. Cheng, M. W. Wu, and I. C. da Cunha Lima, Phys. Rev. B 75, 205328 (2007).
${ }^{17}$ E. Y. Sherman and J. Sinova, Phys. Rev. B 72, 075318 (2005).

${ }^{18}$ I. Tokatly and E. Sherman, Ann. Phys. 325, 1104 (2010).

${ }^{19}$ C. P. Weber, J. Orenstein, B. A. Bernevig, S.-C. Zhang, J. Stephens, and D. D. Awshalom, Phys. Rev. Lett. 98, 076604 (2007).

${ }^{20}$ J. D. Koralek, C. P. Weber, J. Orenstein, B. A. Bernevig, S.-C. Zhang, S. Mack, and D. D. Awschalom, Nature (London) 458, 610 (2009)

${ }^{21}$ J. Wunderlich, A. C. Irvine, J. Sinova, B. G. Park, L. P. Zârbo, X. L. Xu, B. Kaestner, V. Novák, and T. Jungwirth, Nat. Phys. 5, 675 (2009).

${ }^{22}$ A. A. Kiselev and K. W. Kim, Phys. Rev. B 61, 13115 (2000).

${ }^{23}$ M. Ohno and K. Yoh, Phys. Rev. B 77, 045323 (2008).

${ }^{24}$ R. W. Hockney and J. W. Eastwood, Computer Simulation Using Particles (McGraw-Hill, New York, 1981).

${ }^{25}$ C. Jacoboni and L. Reggiani, Rev. Mod. Phys. 55, 645 (1983).

${ }^{26}$ M. V. Fischetti and S. E. Laux, Phys. Rev. B 38, 9721 (1988).

${ }^{27}$ M. V. Fischetti and S. E. Laux, Phys. Rev. B 48, 2244 (1993).

${ }^{28}$ S. Saikin, M. Shen, M.-C. Cheng, and V. Privman, J. Appl. Phys. 94, 1769 (2003).

${ }^{29}$ N. A. Sinitsyn, J. Phys.: Condens. Matter 20, 023201 (2008).

${ }^{30}$ V. Lechner, L. E. Golub, P. Olbrich, S. Stachel, D. Schuh, W. Wegscheider, V. V. Bel'kov, and S. D. Ganichev, Appl. Phys. Lett. 94, 242109 (2009).

${ }^{31} \mathrm{R}$. Winkler, Spin-Orbit Coupling Effects in Two-Dimensional Electron and Hole Systems (Springer, Berlin, 2003).

${ }^{32}$ S. Kettemann, Phys. Rev. Lett. 98, 176808 (2007). 